\title{
INTEGRATING EWOD WITH SURFACE RATCHETS FOR ACTIVE DROPLET TRANSPORT AND SORTING
}

Todd A. Duncombe ${ }^{1}$, Momoko Kumemura ${ }^{2}$, Hiroyuki Fujita ${ }^{2}$, Karl F. Böhringer ${ }^{1}$

${ }^{1}$ Electrical Engineering Department, University of Washington, USA

${ }^{2}$ CIRMM, Institute of Industrial Science, The University of Tokyo, JAPAN
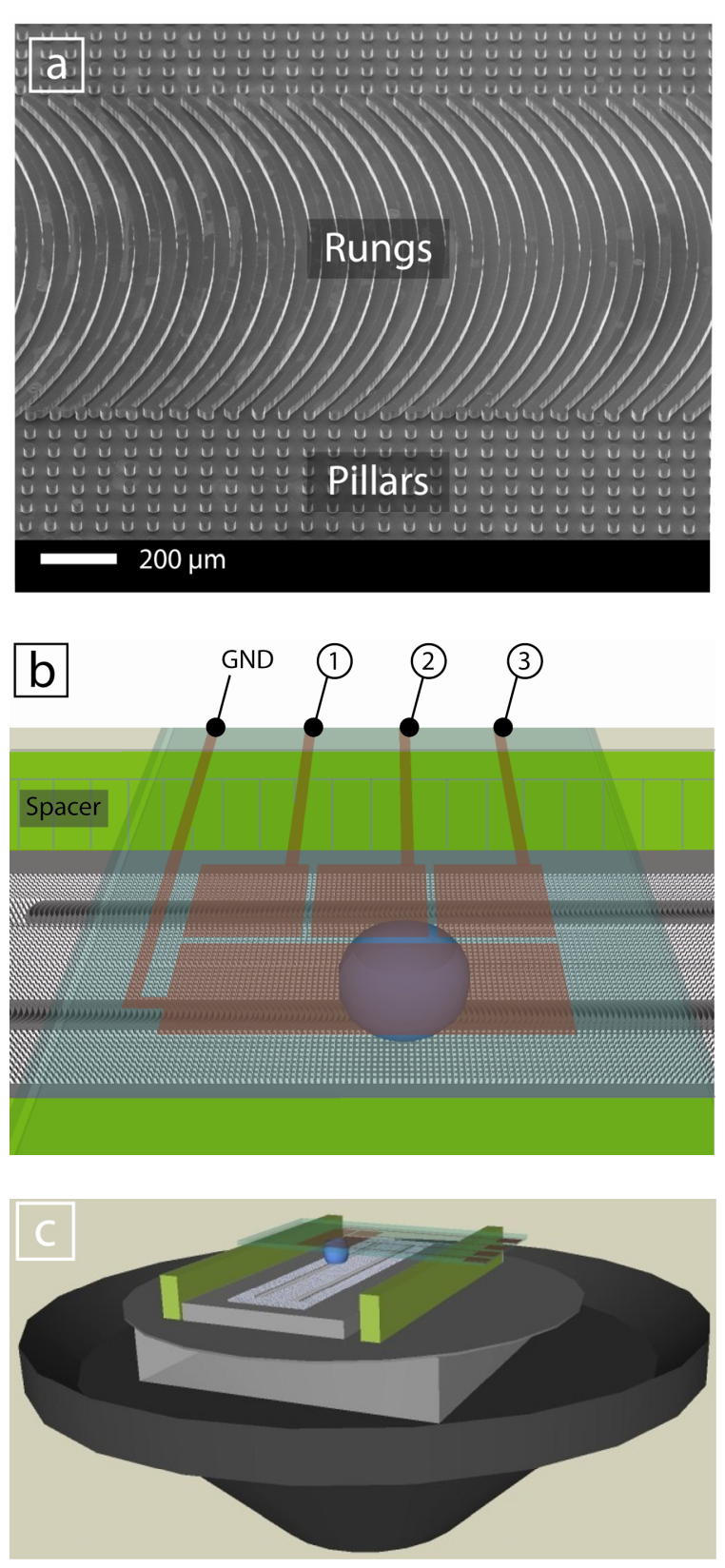

Figure 1: Experimental setup. (a) A SEM micrograph of a surface ratchet. (b) A schematic of the EWOD plate alignment above the surface ratchet, both surfaces contact the droplet. Electrodes 1, 2, 3 and Ground are labeled. (c) The closed system is attached to a stage mounted horizontally on an electromagnetic speaker, which delivers controlled vibrations to the system.

\section{ABSTRACT}

Combining surface ratchets and electrowetting on dielectric (EWOD) produces novel microfluidic systems that achieve passive droplet transport by vibration along microscopically-rough surfaces and active droplet sorting by electric signals. The super-hydrophobic surface ratchet and EWOD plate sandwich a droplet; when vibrated the device adopts passive droplet transportation via the surface ratchet. The EWOD technology is utilized at particular junctions to produce several droplet specific control functions, including the combination of a new ratchet design with an EWOD plate to develop a switch (serving the same purpose as a switch in train tracks) that sorts $10 \mu 1$ droplets at a junction.

\section{INTRODUCTION}

Actuation methods for microfluidic devices designed for transporting discrete liquid volumes have traditionally fallen into two categories; passive droplet transport (global actuation) or active droplet transport (local actuation). There are a wide range of controls for local actuation including light, thermal and electric stimuli. Ichimura et al. [1] developed an active droplet transport device by designing a monolayer of photo-responsive azobenzene groups that altered their wetting properties when exposed to particular wavelengths of light. By applying blue light asymmetrically in intensity on an olive oil droplet, they actively controlled droplet transport. Their surface could then be 'reset' to its original wetting properties with the application of UV light, and droplet transport repeated. Darhuber et el. [2] built a thermocapillary microfluidic device, transporting water droplets by actively engaging micro-heaters in an array, moving a droplet to the adjacent colder region. Similarly to micro-heater arrays, Cho et al. [3] used an array of addressable electrodes for local actuation via the electric wetting properties of polar liquids. Electrowetting on dielectric (EWOD) devices function by reducing the solidliquid surface tension, $\gamma_{\mathrm{SL}}$, of the droplet-surface interface, and thus the droplet is pulled towards the region with high electric field.

Passive droplet transport refers to actuation via a global stimulus. A simple actuation scheme replaces the complex design required for droplet specific control. Physical configuration establishes a bias for motion that drives droplet transport. A classic example is Chaudhury and Whitesides [4] "How to make water run uphill," which presents a passive transport method by varying the chemical composition on a surface to move a droplet against gravity over a limited distance. Dos Santos et al. [5] reported selfpropelling silane droplets on a glass or silicon surface. When transported, droplets alter the chemical composition of the surface thereby requiring extensive cleaning for re- 
peated transports. Bico \& Quéré's recent demonstration of self-propelling bislugs [6] consists of ethylene glycol and silicone oil in a glass capillary. The discrepancy in the capillary pressure drives the motion. Similarly to bislugs, surface ratchets [7] are not limited in transport distance and are repeatable. Surface ratchets use the asymmetric surface contact of a droplet on a rough, hydrophobic surface to achieve transport through vibration. This global actuation transports numerous droplets simultaneously.

\section{DESIGN AND FABRICATION}

Ratchets have a rough, super-hydrophobic surface that creates a biphasic air-solid interface with water, the Fakir state, in which the droplet contacts only the top of the surface's asperities. A droplet in the Fakir state has high apparent contact angles and low resistance during movement [8]. The asymmetric portion of the ratchet is its track, $1.2 \mathrm{~mm}$ in width, which is delimited by a more hydrophobic, rougher region of sparsely spaced pillars (Figure 1A). Pillars have a diameter of $30 \mu \mathrm{m}$ and are spaced in a $70 \mu \mathrm{m}$ pitch. A track is constructed using periodic semicircular rungs ( $650 \mu \mathrm{m}$ in curvature, $30 \mu \mathrm{m}$ width) spaced by $75 \mu \mathrm{m}$. The rung's curvature establishes a discrepancy of surface contact between the two sides of the droplet while the low surface energy of the pillar region contains the droplet on the track. One side of a droplet is aligned with the rung's curvature, giving it uniform surface contact along the rung. The other side is not aligned with the rungs curvature, yielding non-uniform surface contact. When agitated by vibration this discrepancy in edge surface contact drives droplet transport [7].

The microscopically rough surface is fabricated by etching a silicon wafer to a depth of $70 \mu \mathrm{m}$. It is then coated with a monolayer of perfluoro-octyltrichlorosilane (FOTS), deposited from its vapor phase, producing a hydrophobic surface. Vibration is provided by a horizontal stage mounted on an electromagnetic speaker.

EWOD devices are typically designed as a two-plate closed system [3]. To integrate EWOD with surface ratchets we use a one-plate design [9], with grounded electrodes running parallel to control electrodes. The design consists of a $2 \mathrm{~mm} \times 6 \mathrm{~mm}$ rectangular ground electrode running parallel to and spaced $4 \mu \mathrm{m}$ from a series of three 2 $\mathrm{mm} \times 2 \mathrm{~mm}$ control electrodes (control electrodes are separated by $4 \mu \mathrm{m}$ ) (Figure 1B). Each electrode is connected to a contact pad, allowing us to address each electrode individually. We use an AC power source to reduce the risk of electrolysis destroying the electrodes.

The device is constructed on a glass wafer. Fabrication begins with photolithography and a $50 \mathrm{~nm}$ ITO evaporation. A $500 \mathrm{~nm}$ layer of $\mathrm{SiO}_{2}$ is coated using plasma deposition. In the final step the surface is spin-coated with a thin layer of CYTOP, a hydrophobic dielectric layer. During the process, tape is placed over the ITO contact pads during the deposition of $\mathrm{SiO}_{2}$ and CYTOP, preserving an area where electrical connections can be made.

Combining these two technologies, we constructed a device that provides droplet specific control at discrete locations on a surface ratchet. Spacers are used to suspend the
EWOD plate above and face to face with the ratchet. The distance between the two surfaces is chosen such that the droplet contacts both. The surface ratchet, spacer and EWOD plate are placed on a stage mounted horizontally to an electromagnetic speaker, which provides vertical vibrations (Figure 1C). This is the first instance a surface ratchet has been shown functioning contained in a closed system and the first time a single EWOD plate was used to move large $(>10 \mu \mathrm{l})$ droplets while inverted. The ratchets operating conditions require lower frequency and higher amplitude as compared to surface ratchets contacting only one surface.

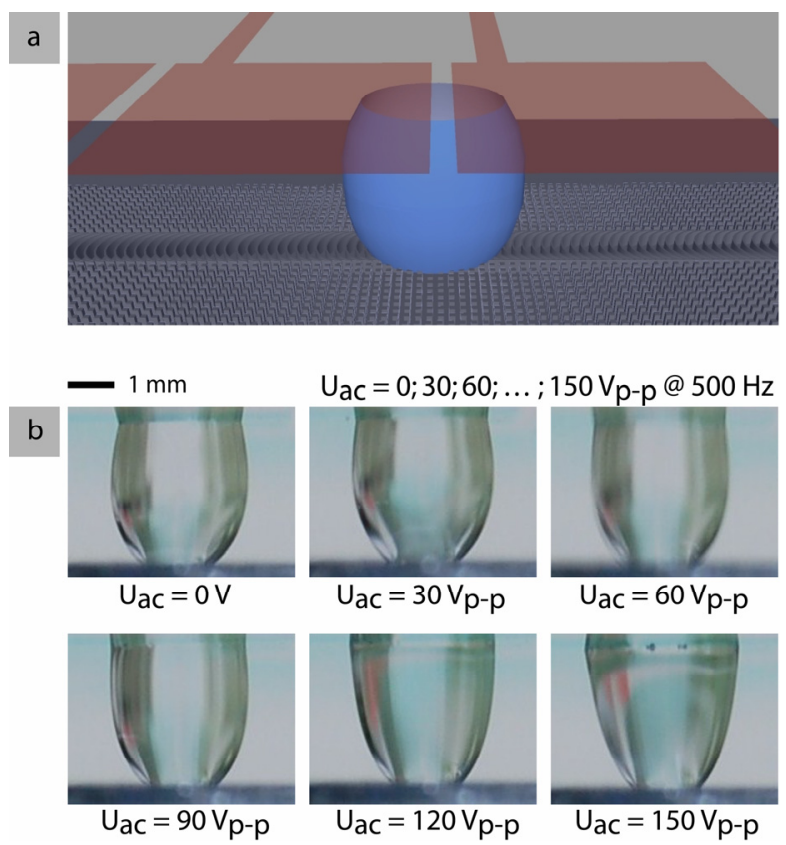

Figure 2: Droplet surface contact vs. applied voltage. (a) A $20 \mu \mathrm{L}$ droplet is placed in between a surface ratchet and EWOD plate spaced by $3.5 \mathrm{~mm}$. (b) The images are taken looking between the two surfaces. As voltage is increased the contact angles with the EWOD plate are reduced, lifting the droplet's center of gravity further from the ratchet.

\section{RESULTS}

When a droplet is under an activated EWOD device, the center of mass of the droplet is lifted up and away from the surface ratchet. The droplet's contact angle with the EWOD plate decreases as its area of contact expands (Figure 2). Increasing the applied voltage increases the electric field and further raises the droplet's center of mass.

Using this novel combination of microfluidic methods we demonstrate three important functions: a valve, a flow reversal device, and a switch for sorting droplets.

\section{Valve}

The most basic function of our system is a valve. Surface ratchet design and vibration cause the droplet to move in the direction of the track's asymmetrical bias. By activating electrodes placed at specific locations we are able to 
stop an advancing droplet and hold it in place as the system continues to vibrate. When the electrodes are deactivated the droplet resumes movement along the track.
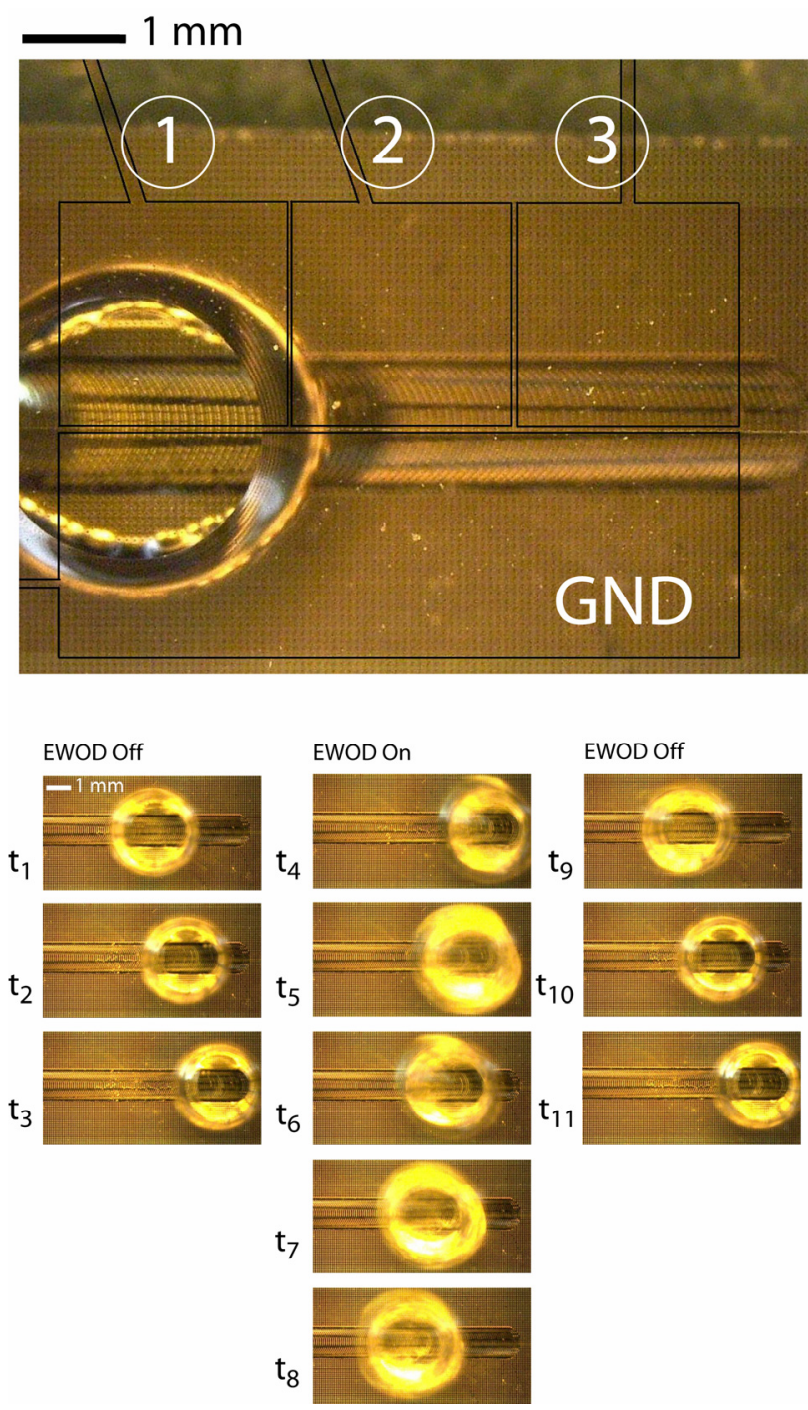

Figure 3: Flow Reversal Device. A $20 \mu L$ droplet is vibrated by the electromagnetic speaker at $4.2 \mathrm{~V}$ and $32 \mathrm{~Hz}$; $150 \mathrm{~V}$ at $500 \mathrm{~Hz}$ is applied to the electrodes. In frames $t_{1}$ $t_{3}, E W O D$ is off and the droplet advances via the surface ratchet at an average pace of $3 \mathrm{~mm} / \mathrm{s}$. At $t_{4}$ electrode 3 is activated (1, 2 float). Droplets appear larger when affected by the EWOD device due to the increased contact with the EWOD plate. At $t_{5}$ electrode 2 is activated, stretching the droplet to the left. At to electrode 3 is deactivated and the droplet is pulled to the left, centering itself on electrode 2. The process in $t_{4}-t_{6}$ is repeated in $t_{6}-t_{8}$ with electrodes 1 and 2, at the droplet has moved backwards returning to the original position in $t_{1}$. When EWOD is disengaged, in $t_{9}-t_{11}$, the droplet returns to its initial contact angles and resumes motion via the surface ratchet.
Figure 2A shows our implementation of the valve. The minimum voltage required to stop the droplet must be large enough to reduce the droplet's contact with the surface ratchet such that the adhesion force due to EWOD is stronger than the force from the track's bias. To stop and hold a $20 \mu \mathrm{l}$ droplet advancing along the track the minimum applied voltage is $65 \mathrm{~V}$ at $500 \mathrm{~Hz}$.

\section{Flow Reversal Device}

The second function we developed is a flow reversal device. The flow reversal device transports droplets in the opposite direction from the ratchet's asymmetrical bias while the stage continues to vibrate. Figure 3 outlines the electrode placement above the ratchet. One ground electrode and three control electrodes are placed over the surface ratchet. By sequentially activating and deactivating electrodes we are able to stop an advancing droplet and reverse its direction. We use a higher voltage $(150 \mathrm{~V}$ at 500 $\mathrm{Hz}$ ) in the flow reversal device than the valve to produce rapid and reliable transport.

The operation of the flow reversal device involves multiple stages. Electrode 3 in Figure 3 is activated while the other two electrodes are floating. Initially like a valve, the advancing droplet is stopped at electrode 3. At this point, electrode 2 is activated. The resulting electric field stretches the droplet against track's bias (as seen between $t_{4}-t_{5}$ ) thus aligning the droplet between electrodes 2 and 3 . Electrode 3 is then deactivated and the droplet moves backwards $\left(t_{5}-t_{6}\right)$ to center itself on electrode two. This sequence is then repeated to move a droplet backwards any desired distance.

\section{A Switch (Sorting Droplets)}

We demonstrate a switch function that enables droplet specific sorting using EWOD with a novel surface ratchet design (Figure 4). The new ratchet design is a four way intersection of tracks; one track's asymmetric bias is towards the junction while the other three are away from it. The EWOD switch at the junction transports droplets against the track's asymmetrical bias to a position over a new track oriented in a direction perpendicular to the incoming track. After the droplet has been moved, the electrodes are deactivated and the droplet resumes ratchet driven motion in the chosen direction.

Electrodes are controlled to turn a droplet left, right or let it pass straight through. As seen in Figure 4's electrode configuration, electrodes are above the right hand and left hand path. When one of these electrodes is activated, an advancing droplet will be pulled by the electric field as it reaches the junction against the track's bias. If the voltage level is sufficient, the droplet will center itself under the activated electrode. The track's bias under these electrodes favors movement along the new track's direction. Thus, when the electrode is deactivated the droplet begins movement via the surface ratchet in its new direction. If both electrodes are deactivated the droplet is unaffected by the junction and continues straight along the ratchet. 


\section{DISCUSSION}

Active and passive droplet transport methods have their own independent applications, hinging on whether droplet specific control is required or not. A limitation of passive transportation is the difficulty to address and guide specific droplets. For surface ratchets, or any other passive droplet transportation method, to be useful in a wide range of microfluidic applications, a method of active, droplet-specific transport is essential.

We have implemented a device that integrates the two, taking advantage of surface ratchet's passive transport characteristics, and EWOD's droplet specific control. Using the integrated system, we have demonstrated three critical functions that could greatly expand the applications of surface ratchets. Now a device that functions primarily through surface ratchet's passive droplet transportation, moving several droplets over long periods of time, can be controlled at specific junctions, stopping droplet flow with a valve, moving droplets backwards with a flow reversal device, and sorting droplets at a switch.

\section{ACKNOWLEDGEMENTS}

This work was supported by an International Research and Education in Engineering (IREE) supplement to National Science Foundation grant ECCS-05-01628 (Rajinder Khosla, program director). The authors thank VLSI Design and Education (VDEC) for mask production, Laurent Jalabert, Cagatay Tarhan, Bernard Wee, Christophe Yamahata and all the members of CIRMM at the University of Tokyo for their generous help and support.

\section{REFERENCES}

[1] Kunihiro Ichimura, Sang-Keun Oh, Masaru Nakagawa. "Light-Driven Motion of Liquids on a Photoresponsive Surface." Science 288(547):1624-1626, 2000.

[2] Anton A. Darhuber, Joseph P. Valentino, Sandra M. Troian, and Sigurd Wagner. "Thermocapillary Actuation of Droplets on Chemically Patterned Surfaces by Programmable Microheater Arrays." Journal of Microelectromechanical Systems 12(6), 2003.

[3] S. K. Cho, H. Moon and C.-J. Kim, "Creating, Transporting, Cutting, and Merging Liquid Droplets by Electrowetting-Based Actuation for Digital Micro-fluidic Circuits," Journal of Microelectromechanical Systems 12(1):70-80, 2003.

[4] Chaudhury, M.K. and G.M. Whitesides, "How to make water run uphill?" Science 256(5063):1539-1541, 1992.

[5] Fabrice Domingues Dos Santos and Thierry Ondarcuhu. "Free-Running Droplets." Physical Review Letters 75(16), 1995.

[6] J. Bico \& D. Quéré, "Self-propelling slugs in a tube", J. Fluid Mech. 467(201), 2002.

[7] A. Shastry, D. Taylor and K. F. Böhringer, "MicroStructured Surface Ratchets for Droplet Transport," in Transducers'07, pp. 1353-1356, Lyon, France, 2007.

[8] Cottin-Bizonne, C., et al., "Low-friction flows of liquid at nanopatterned interfaces." Nature Materials 2:237240, 2003.

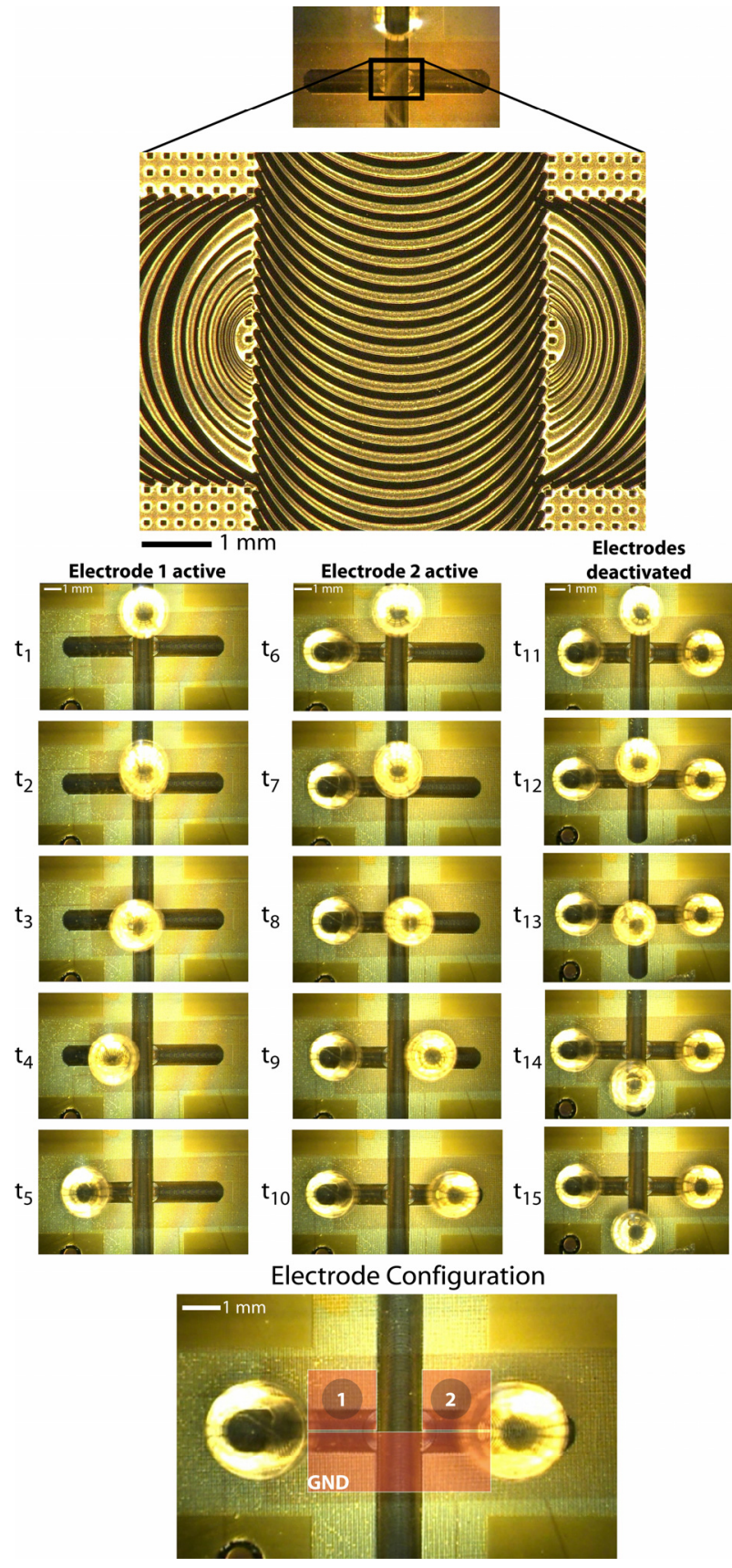

Figure 4: Sorting droplets. Depending whether electrode 1 or 2 is activated, the droplet turns to the left or right, respectively. A $10 \mu \mathrm{L}$ droplet is vibrated at $8 \mathrm{~V}$ and $55 \mathrm{~Hz}$, the applied voltage at the electrodes is $150 \mathrm{~V}$ at $500 \mathrm{~Hz}$. In $t_{1}-t_{2}$ the droplet advances using the surface ratchet. In $t_{3}-t_{4}$ the droplet 'feels' the electric field and is pulled towards electrode 1 . At $t_{5}$ the electrode is turned off and the droplet resumes its initial contact, and moves in the new direction. This procedure is repeated with electrode 2 in $t_{6}$ - $t_{10}$ to sort droplets in the alternate direction. In $t_{11}-t_{15}$ the electrodes are deactivated; droplets are unaffected by the junction.

[9] U.-C. Yi and C.-J. Kim, "EWOD actuation with electrode-free cover plate," in Transducers'05, pp. 89- 92, Seoul, Korea, 2005. 